\title{
Asociación de tiburones con el Monte Submarino Las Gemelas y primera evidencia de conectividad con la Isla del Coco, Pacífico de Costa Rica
}

\author{
Elpis J. Chávez ${ }^{1 *}$, Randall Arauz ${ }^{2,4}$, Alex Hearn $^{3,4,5}$, Elena Nalesso ${ }^{1} \&$ Todd Steiner ${ }^{3,4}$ \\ 1. Centro Rescate de Especies Marinas Amenazadas CREMA. 1.5 Km N, Calle 114, Carretera a San José de la Montaña, \\ 40201, Barva, Heredia, Costa Rica; echavk@gmail.com; nalesso.elena@gmail.com \\ 2. Fins Attached Marine Research and Conservation. 19675 Still Glen Drive Colorado Springs 80908, Colorado, USA; \\ rarauz@finsattached.org \\ 3. Turtle Island Restoration Network. Forest Knolls 94933, California, USA; tsteiner@tirn.net \\ 4. MigraMar. Mercaderes 130 San José Insurgentes, 03900, Benito Juárez, Ciudad de México, México. \\ 5. Galapagos Science Center, Universidad San Francisco de Quito, Diego de Robles y Vía Interoceánica, Quito, Ecuador. \\ ahearn@usfq.edu.ec \\ * Correspondence
}

Recibido 30-I-2019. Corregido 28-IV-2019. Aceptado 16-VI-2019.

\begin{abstract}
Association of sharks with Las Gemelas Seamount and first evidence of connectivity with Cocos Island, Pacific of Costa Rica. Introduction: Seamounts and oceanic islands are known as hotspots of pelagic biodiversity, which highly migratory species use as natural biological corridors. Although marine protected areas have been established in the Eastern Tropical Pacific with a goal to protect and manage region's marine biodiversity, not all utilize or enforce a no-take policy, and none are capable of protecting highly mobile species once they move outside of the limit of these areas. Objective: We present the first evidence of shark connectivity between Cocos Island National Park (CINP) and Las Gemelas seamount located in the Seamounts Marine Management Area (SMMA), by a female scalloped hammerhead shark (Sphyrna lewini). Methods: Between May 2015 and May 2016 a scalloped hammerhead shark and a pelagic thresher shark (Alopias pelagicus) were tagged (V16, Vemco Ltd.) and monitored by an array of acoustic receivers installed at CINP and Las Gemelas. We analyzed the acoustic data descriptively and we calculated the residency index (IR). Results: $S$. lewini performed persistent movements between these two marine protected areas. All detections in Cocos Island occurred during the morning (7:00-12:00 h) and Roca Sucia was the most visited site. The shark shows a low residency $(\mathrm{IR}=0.02)$ and intermittent presence in the study area. We recorded the presence of A. pelagicus at Las Gemelas almost one year after it was tagged at the site. Conclusions: The persistent movements of S. lewini between these two pelagic marine protected areas, and the presence of a tagged A. pelagicus at Las Gemelas seamount, suggests that movements between oceanic islands in the region may be more complex than simple "back and forth" movements, with seamounts in between them acting as stepping stones, where they may also stay for periods of time. Our results emphasize the importance of granting greater protection to seamounts and creating connecting swimways to oceanic island hotspots for the conservation of pelagic and highly migratory species. Although the establishment of marine reserves around oceanic islands and seamounts have been proposed as an effective way to conserve and protect marine biodiversity in the high seas, their effectiveness is only partial. This study highlights the importance of studying the spatial dynamics of highly migratory species to help improve the design and efficiency of marine protected areas located in the high seas such as CINP and SMMA.
\end{abstract}

Key words: Eastern Tropical Pacific, highly migratory species, Sphyrna lewini, Alopias pelagicus, Marine Protected Areas.

Chávez, E. J., Arauz, R., Hearn, A., Nalesso, E., \& Steiner, T. (2020). Asociación de tiburones con el Monte Submarino Las Gemelas y primera evidencia de conectividad con la Isla del Coco, Pacífico de Costa Rica. Revista de Biología Tropical, 68(Supl. 1), S320-S329. 
Las islas oceánicas y los montes submarinos son conocidos como "puntos calientes" o "hotspots" de biodiversidad marina, en especial de especies pelágicas (Worm, Lotze, \& Myers, 2003; Morato, Hoyle, Allain, \& Nicol, 2010). Dicha formaciones se caracterizan por presentar condiciones oceanográficas y topográficas locales que promueven el transporte y la circulación de nutrientes favoreciendo así la productividad, la biomasa y la diversidad de especies (Worm et al., 2003; Lavelle \& Mohn, 2010; Lizano, 2012). Al ser áreas clave para la alimentación y el descanso de especies pelágicas altamente migratorias, estos sitios han sido considerados como corredores biológicos naturales (Klimley \& Nelson, 1984; Klimley, 1987; Pitcher et al., 2008; Hearn, Ketchum, Klimley, Espinoza \& Peñaherrera, 2010; Heidemeyer, 2015). Dada la importancia biológica de estas formaciones ubicadas en aguas abiertas, el garantizar su protección puede ser una manera efectiva de lograr la conservación de varias especies a la vez (Worm et al., 2003). A lo largo del Pacífico Tropical Oriental (PTO), se han establecido una serie de reservas marinas ubicadas alrededor de las diversas islas, archipiélagos y montes submarinos que conforman el Corredor Marino del Pacífico Tropical Oriental (CMAR) como Malpelo y Gorgona (Colombia), Isla del Coco (Costa Rica), Coiba (Panamá) e islas Galápagos (Ecuador). El objetivo principal de dichas reservas marinas es proteger y manejar los recursos marinos que se encuentran dentro de esta región (Meneses, 2005; Rothschild, 2005).

Desafortunadamente, los montes submarinos han sido históricamente codiciados y sobreexplotados por las pesquerías de todo el mundo (Rogers, 1994). En Costa Rica, tanto la Isla del Coco como los montes submarinos adyacentes han sido blanco de la explotación pesquera por parte de flotas nacionales y extranjeras (Lizano, 2012; Rodríguez \& Rosero, 2018). Por lo que, la presión pesquera (legal e ilegal) existente en estos sitios constituye una de las principales amenazas a su integridad ecológica (Arias, Pressey, Jones, Álvarez-Romero, \& Cinner, 2016; Rodríguez
\& Rosero, 2018). Aunque dicha actividad está enfocada principalmente a la captura de atún (Scombridae) y dorado (Coryphaena hippurus), los tiburones de las familias Carcharhinidae, Alopiidae y Sphyrnidae suelen constituir una parte importante de las capturas (Dapp, Arauz, Spotila \& O’Connor, 2013; LópezGarro, Zanella, Martínez, Golfín-Duarte, \& Pérez-Montero, 2016; Rodríguez \& Rosero, 2018; Nalesso et al., 2019).

Diversos estudios han documentado la conectividad entre las poblaciones de tiburones y tortugas del CMAR y las zonas costeras de Centroamérica (Bessudo et al., 2011; Ketchum et al., 2014a; Nalesso et al., 2019; Heidemeyer, 2015; MigraMar, 2019). Dicha conectividad resalta la importancia que tienen estas estas islas en la supervivencia de las especies altamente móviles o migratorias. Sin embargo, todavía existe muy poca información sobre el papel que juegan los montes submarinos en la conservación de especies amenazadas de extinción como los tiburones. Este estudio presenta la primera evidencia de asociación de un tiburón zorro (Alopias pelagicus) con el monte submarino Las Gemelas, y la primera evidencia de conectividad espacial de éste monte submarino con la Isla del Coco, ubicada $\sim 50$ $\mathrm{km}$ al noreste, por parte de un tiburón martillo (Sphyrna lewini).

\section{MATERIALES Y MÉTODOS}

Área de estudio: El Parque Nacional Isla del Coco (PNIC) se ubica a $550 \mathrm{~km}$ al suroeste de la costa pacífica de Costa Rica y comprende el área terrestre de la Isla del Coco $\left(23.85 \mathrm{~km}^{2}\right)$, así como sus aguas circundantes en un radio de $22.2 \mathrm{~km}$ (Cajiao, 2008) abarcando una extensión marina de protección absoluta de 1989 $\mathrm{km}^{2}$. Por su parte, la recientemente creada Área Marina de Manejo Montes Submarinos (AMMMS) consiste en un rectángulo de $9649 \mathrm{~km}^{2}$ de extensión ubicado en las aguas circundantes al PNIC, comprendiendo la porción noreste y suroeste de la Cordillera Volcánica del Coco $(\mathrm{CVC})$. De acuerdo con la zonificación del AMM-MS existen dos zonas de protección 
absoluta, una que consiste de una "ceja" de 742 $\mathrm{km}^{2}$ que colinda con el límite Noreste del PNIC y que actúa como zona de amortiguamiento del PNIC (SINAC, 2013), y otra de $2733 \mathrm{~km}^{2}$ en el extremo suroeste que se ubica sobre los montes submarinos "Las Gemelas", cuyas cimas se encuentran entre 150 y $180 \mathrm{~m}$ de profundidad respectivamente (Cajiao, 2008; Decreto ejecutivo $N^{\circ}$ 36452-MINAET, 2011; Rojas \& Alvarado, 2012; SINAC, 2013) (Fig. 1).

Marcaje y monitoreo: El 8 de mayo del 2015 se colocó un transmisor acústico externo (V16, Vemco Ltd.; ID 22437, $158 \mathrm{~dB}$, transmisión aleatoria entre $40-80 \mathrm{~s}$; duración de la batería $~ 3.7$ años) en una hembra de $S$. lewini de aproximadamente $250 \mathrm{~cm}$ de longitud total (LT) en el sitio conocido como Roca Sucia, ubicado al noroeste de la Isla del Coco. El transmisor acústico consiste de un cilindro de PVC con un diámetro de $1.6 \mathrm{~cm} \mathrm{x} 7 \mathrm{~cm}$ de largo que emite una señal acústica a $69 \mathrm{kHz}$ el cual fue atado a un dardo de acero inoxidable mediante un cable de acero inoxidable de 12 $\mathrm{cm}$ de largo. El dardo se fijó al tejido muscular del tiburón ubicado debajo de la primera aleta dorsal mediante un arpón modificado durante la realización de buceo SCUBA, de manera que el transmisor quedó colgando externamente.

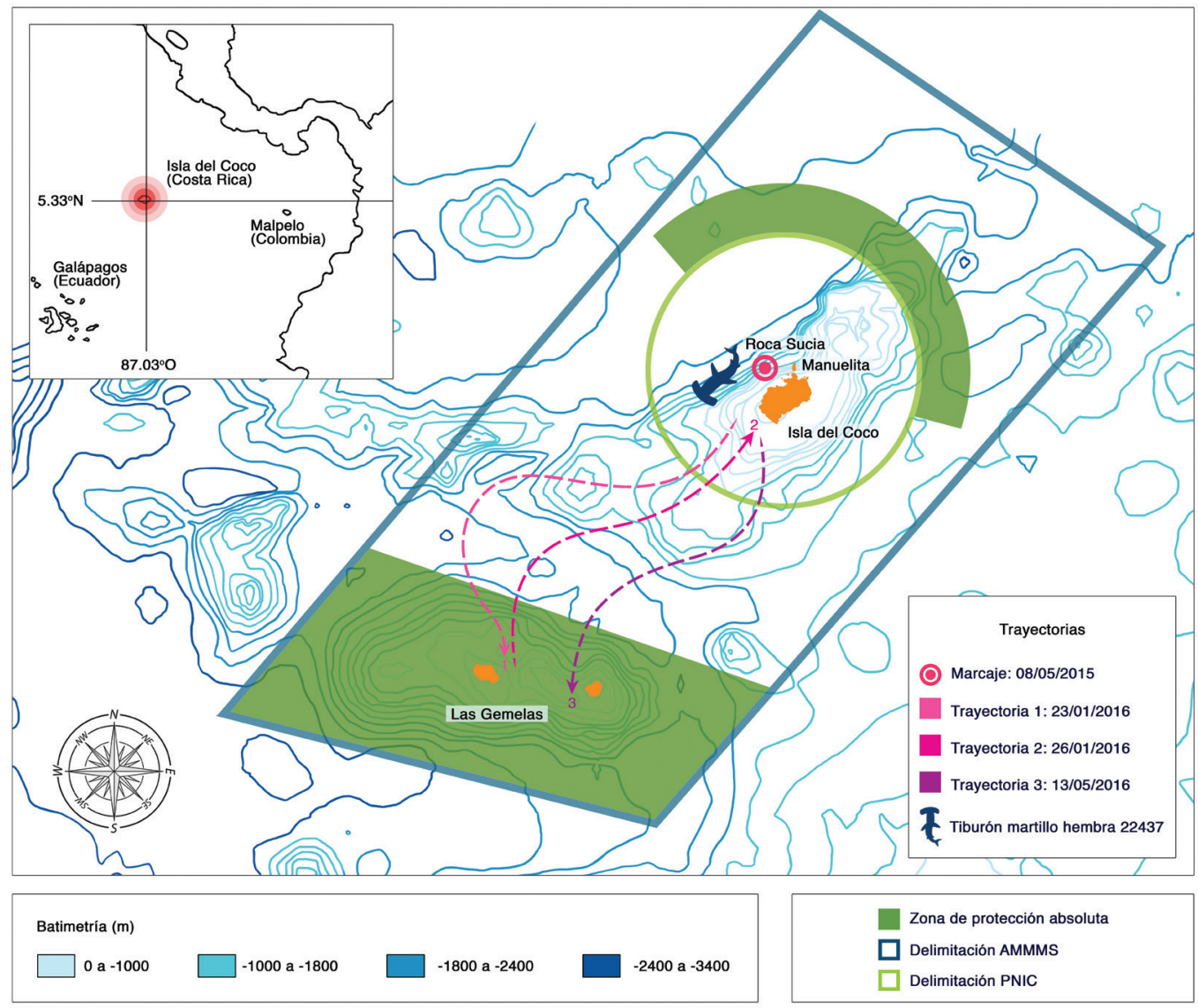

Fig. 1. Movimientos del tiburón martillo ID 22437 entre el Parque Nacional Isla del Coco (PNIC) y el Área Marina de Manejo Montes Submarinos (AMM-MS) durante mayo del 2015 y mayo del 2016. Las líneas discontinuas sugieren la posible trayectoria realizada por el tiburón.

Fig. 1. Movements of scalloped hammerhead shark ID 22437 between Cocos Island National Park (CINP) and the Seamounts Marine Management Area during May 2015 and May 2016. Dotted lines suggest the potential shark's trajectory. 
Los movimientos del tiburón marcado fueron registrados mediante una red de siete receptores acústicos (VR2W, Vemco Ltd.) instalados en puntos estratégicos alrededor de la Isla del Coco donde se conoce que ocurre la agregación de tiburones martillo adultos, específicamente en los alrededores del islote Manuelita, Ulloa, Alcyone, Roca Sucia, Dos Amigos Pequeño y Punta María (ver Nalesso et al., 2019). Dos receptores acústicos adicionales (VR2AR, Vemco Ltd.) fueron instalados el cinco de mayo del 2015 a 180 y 190 m de profundidad en diferentes cumbres del monte submarino conocido como Las Gemelas, localizado aproximadamente a $50 \mathrm{~km}$ al suroeste de la Isla del Coco (Starr, Green, \& Sala, 2012). Para garantizar la correcta instalación de los receptores se recurrió al uso de sumergibles tripulados y equipados con brazos mecánicos. Las pruebas para determinar el rango de detección acústica de los receptores en la Isla del Coco estimaron un alcance de entre 150 y $300 \mathrm{~m}$ (Nalesso et al., 2019). Los receptores acústicos alrededor de Isla del Coco fueron recuperados y reinstalados cada seis meses por buzos, mientras que los dos receptores en Las Gemelas fueron recuperados al cabo de 15 meses mediante una señal acústica que activó el sistema de liberación. Una vez liberados los receptores fueron transportados hacia la superficie por medio de boyas. Desafortunadamente, uno de los receptores acústicos no pudo ser recuperado, probablemente debido a alguna falla en el sistema de flotación. Los datos acústicos de todos los receptores fueron descargados a una base de datos electrónica administrada por MigraMar.

Durante la expedición a Las Gemelas, se realizó una jornada de pesca de investigación entre las 15:00 y las 21:00 hrs, utilizando un palangre corto modificado $(300 \mathrm{~m}$ de línea madre, 20 anzuelos circulares \#14 sin barba), que resultó en la captura de un tiburón zorro pelágico macho (Alopias pelagicus) de 236 $\mathrm{cm}$ de LT, el cual fue equipado con un transmisor acústico interno (V16, Vemco Ltd.; ID 32359; $158 \mathrm{~dB}$; transmisión aleatoria entre 120 - 230s; duración de la batería 3.2 años). En este caso, se procedió a realizar una pequeña incisión quirúrgica en el vientre del tiburón para insertar el transmisor en la cavidad peritoneal. Todos los métodos utilizados durante esta investigación fueron aprobados $\mathrm{y}$ realizados bajo los permisos de investigación ACMICI-2015-008 y 2016-I-ACMIC-07 otorgados por el Área de Conservación Marina Cocos (SINAC-MINAE).

Análisis de datos: Los datos de presenciaausencia del tiburón martillo alrededor de los receptores fueron analizados de manera descriptiva. El tiburón se consideró presente en el área de estudio cuando dos o más detecciones fueron registradas durante el mismo día por cualquiera de los receptores. Los días y sitios de detección fueron graficados en una línea de tiempo para determinar los movimientos, sitios visitados y las posibles trayectorias realizadas. Además, se calculó el índice de residencia en el área de estudio (IR), dividiendo el número de días en que el tiburón fue detectado por cualquiera de los receptores entre el número total de días de monitoreo (Espinoza, Heupel, Tobin, \& Simpfendorfer, 2015). Debido a que la red de receptores no cubría toda el área del PNIC y el AMM-MS, el índice de residencia es un valor mínimo aproximado del tiempo que permaneció el tiburón dentro del área de estudio, ya que el tiburón pudo estar presente en la Isla del Coco o en los montes submarinos sin ser detectado por los receptores acústicos (Nalesso et al., 2019).

\section{RESULTADOS}

Desde la fecha de su marcaje el 8 de mayo del 2015 el tiburón martillo ID 22437 fue detectado 139 veces de manera intermitente por los receptores de la Isla del Coco y Las Gemelas hasta mayo del 2016, las cuales nunca ocurrieron por más de tres días consecutivos. Los datos acústicos muestran que el tiburón martillo permaneció en los alrededores de la Isla del Coco durante siete días después de su marcaje en Roca Sucia. Durante este tiempo el tiburón visitó repetidamente Roca Sucia y realizó un desplazamiento a Manuelita, para 


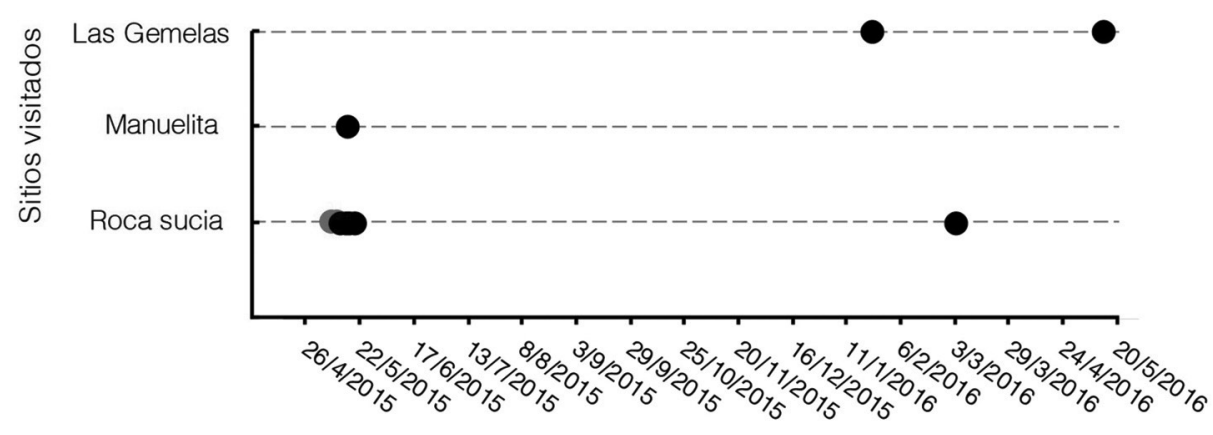

Periodo de monitoreo

Fig. 2. Registro de presencia-ausencia del tiburón martillo ID 22437 en los sitios ubicados dentro del PNIC y el AMM-MS, entre mayo 2015 y mayo 2016. El punto gris indica la fecha de marcaje del tiburón.

Fig. 2. Presence-absence of scalloped hammerhead shark ID 22437 at sites located at Cocos Island National Park (CINP) and Seamounts Marine Management Area, during May 2015 and May 2016. The gray dot indicates tagging date.

regresar un día después (Fig. 1 y 2). Después de ocho meses de ausencia, se registró la presencia del tiburón martillo por primera vez en Las Gemelas el 23 enero del 2016. Tres días más tarde el tiburón regresó a la Isla del Coco, donde fue detectado nuevamente por el receptor de Roca Sucia. El tiburón fue detectado por última vez 28 días más tarde en Las Gemelas el 13 mayo 2016 (Fig. 1 y 2). El índice de residencia de $S$. lewini en el área de estudio fue de 0.02. Roca Sucia fue el sitio más visitado por el tiburón desde su marcaje, con un total de 114 detecciones, en comparación con 6 en Manuelita y 19 en Las Gemelas. Todas las detecciones ocurrieron entre las 7:00 y las 12:00 horas. Adicionalmente, se registró la presencia un tiburón zorro pelágico en Las Gemelas el 18 de marzo del 2016, casi un año después de su marcaje (5 mayo del 2015). $A$. pelagicus permaneció durante cinco días más en este monte submarino, siendo detectado un total de 30 veces.

\section{DISCUSIÓN}

El presente estudio constituye la primera evidencia de asociación entre un tiburón zorro con el monte submarino Las Gemelas, y el primer reporte de conectividad espacial entre éste y el PNIC por parte de un tiburón martillo. Se ha propuesto que los tiburones y otras especies marinas son capaces de utilizar diversas pistas ambientales, químicas y/o magnéticas para orientarse y navegar a través del océano (Lohmann, Lohmann, \& Endres, 2008; Nosal, Chao, Farrara, Chai, \& Hastings, 2016). Es posible que el tiburón martillo se haya desplazado a través de la Cordillera Volcánica del Coco utilizando las anomalías magnéticas de estos sitios para orientarse durante su viaje (Klimley, 1993).

Todas las detecciones acústicas del tiburón martillo ID 22437 ocurrieron únicamente durante la mañana, lo cual es congruente con estudios que han demostrado que $S$. lewini es una especie altamente gregaria que tiende a formar grupos alrededor de islas y montes submarinos en aguas relativamente someras (25-30 m) principalmente durante el día (Klimley \& Nelson, 1984). Se ha propuesto que la formación de grandes grupos de $S$. lewini alrededor de estos sitios puede ser una estrategia para optimizar su energía, antes de emprender el viaje hacia aguas profundas durante la noche probablemente para alimentarse (Klimley \& Nelson, 1984; Holland, Wetherbee, Peterson, \& Lowe, 1993; Bessudo et al., 2011; Ketchum et al., 2014b).

La baja residencia y los largos periodos de ausencia exhibidos por el tiburón martillo ID 22437 muestran que este tiburón utilizó de manera temporal la Isla del Coco y el 
monte submarino Las Gemelas. Los periodos de ausencia (e. i. días, semanas o meses) han sido observados en otras islas y montes submarinos del PTO, lo cual hace pensar que se trata de un comportamiento común en la especie (Klimley \& Nelson, 1984; Bessudo et al., 2011; Nalesso, 2014; Nalesso et al., 2019; Ketchum et al., 2014a). Basados en datos provenientes de 84 tiburones martillo monitoreados entre 2005 - 2013, Nalesso et al. (2019) encontraron que los tiburones martillo de la Isla del Coco no permanecen de manera constante en la isla, sino que estos suelen ausentarse por periodos de nueve meses o más. Aunque la ausencia de detecciones durante varios meses a la vez podría estar relacionada con el desplazamiento del tiburón a lugares de la isla donde no había receptores instalados, es muy probable que el tiburón martillo ID 22437 haya realizado viajes a lugares alejados, como aguas continentales $\mathrm{u}$ otras islas del CMAR. Diversas investigaciones han planteado que $S$. lewini podría estar utilizando las islas o montes submarinos como puntos de referencia durante sus migraciones diarias o estacionales (Klimley \& Nelson, 1984; Hearn, et al., 2010; Ketchum et al., 2014a; Hoyos-Padilla, Ketchum, Klimley \& Galván-Magaña, 2014). Los movimientos de ida y vuelta realizados por $S$. lewini entre las islas Malpelo, Cocos y Galápagos ya han sido debidamente documentados en varios estudios (Bessudo et al., 2011; Ketchum et al., 2014a; Nalesso et al. 2019). Estos movimientos entre islas sugieren que las poblaciones de Galápagos y Malpelo pueden estar utilizando la Isla del Coco, y potencialmente Las Gemelas como puntos de referencia durante sus migraciones estacionales a zonas costeras de Centroamérica y Sudamérica (Nalesso et al. 2019). Recientemente se ha sugerido que las especies altamente migratorias utilizan la Cordillera Volcánica del Coco como una "vía" o autopista para trasladarse entre los sitios de agregación y alimentación ubicados en aguas abiertas del PTO (Peñaherrera-Palma et al. 2018).

La residencia temporal de los tiburones martillo en el PNIC podría estar relacionada con sitios de alimentación, de refugio, estaciones de limpieza, o con eventos reproductivos (Hamilton \& Watt, 1970; Clarke, 1971; Klimley \& Nelson, 1984; Dewar et al., 2008; Bessudo et al., 2011; Oliver, Hussey, Turner, \& Beckett, 2011; Torres-Rojas et al., 2013; Ketchum et al., 2014a). Hasta la fecha solo ha sido reportado un evento de apareamiento en la Isla del Coco (Salinas de León, Hoyos-Padilla, \& Pochet, 2017). Investigaciones realizadas por SibajaCordero (2008) y Nalesso (2014) han señalado que los tiburones martillo en la Isla del Coco suelen permanecer o regresar continuamente a sitios como Alcyone, Roca Sucia y Manuelita donde existen estaciones de limpieza permanentes. Por lo tanto, los movimientos entre Manuelita y Roca Sucia, pueden estar impulsados probablemente por la disponibilidad de espacio en las estaciones de limpieza ubicadas en estos sitios.

A pesar de los esfuerzos de investigación realizados en los últimos años, aún existe muy poca información sobre la fauna que habita en los montes submarinos y sobre su relación con otros montes submarinos, islas oceánicas y zonas costeras (Rogers, 1994; Cortés \& Blum, 2008; Cortés et al., 2012). Aunque los movimientos de un solo individuo pueden no ser una representación fiel del comportamiento de toda la población, este hallazgo recalca la importancia de seguir investigando las posibles interconexiones ecológicas de los montes submarinos con la Isla del Coco y su relación con otras islas del PTO (Cortés \& Blum, 2008). Si bien la conectividad entre las poblaciones de $S$. lewini de las islas Malpelo, Cocos y Galápagos ha sido demostrada en varios estudios (Bessudo et al., 2011; Ketchum et al., 2014a; Nalesso et al., 2019), hasta el momento no se había obtenido evidencia acerca de la conectividad entre las poblaciones de estas islas oceánicas y los montes submarinos adyacentes.

Adicionalmente, la presencia periódica de especies amenazadas de extinción como $A$. pelagicus y $S$. lewini en Las Gemelas confirma la importancia de estos montes submarinos para las especies altamente móviles que convergen en estos hábitats pelágicos (Wehrtmann \& Cortés, 2009; Morato et al., 2010). A. pelagicus es 
una especie mayormente oceánica que habita en aguas profundas tropicales y subtropicales del Pacífico e Indo-Pacífico y de la cual se conoce muy poco sobre su biología y ecología (Reardon, Márquez, Trejo, \& Clarke, 2009). Al ser sitios conocidos por su alta productividad y disponibilidad de presas, es probable que $S$. lewini y A. pelagicus hayan coincidido en el monte submarino Las Gemelas para alimentarse. Sin embargo, dado el poco conocimiento que se tiene sobre la dinámica ecológica de estos sitios, sería indispensable recabar mayor información que nos ayude a fundamentar o rechazar esta hipótesis.

Tanto A. pelagicus como S. lewini son importantes componentes de la pesca artesanal y comercial, lo cual ha contribuido de manera importante al declive de sus poblacionales a escala mundial (Baum et al. 2009; Zanella, López-Garro, \& Arauz, 2009; Reardon et al., 2009; Hearn, Utreras, \& Henderson, 2010; White, Myers, Flemming, \& Baum, 2015). Entre las estrategias más recomendadas para lograr la conservación de las especies marinas están aquellas que logran minimizar las interacciones con pesquerías (Nalesso et al., 2019). En este sentido, la creación de AMPs con políticas de protección estricta alrededor de las islas y montes submarinos se ha propuesto como una estrategia para conservar y proteger la biodiversidad marina en aguas abiertas (Worm et al., 2003). Sin embargo, la efectividad de dichas AMPs sigue siendo cuestionada, principalmente por la falta de información científica acerca de las especies que se desea proteger, así como por la carencia de recursos económicos disponibles para su manejo y protección (Hearn et al. 2010; White et al., 2015; Arias et al., 2016).

Aunque el AMM-MS posee zonas de protección absoluta donde se prohíben las actividades pesqueras, estas aún son permitidas en el resto del área marina (Fig. 1). Durante sus recorridos entre el PNIC y Las Gemelas, el tiburón martillo se desplazó más de $30 \mathrm{~km}$ a través de aguas donde se permite la pesca, incrementando así el riesgo de ser capturado por las flotas pesqueras que faenan en esta área. Lo anterior, resalta la necesidad de contar con mejor información sobre la distribución y dinámica espacial de especies altamente móviles que nos permita evaluar y proponer esquemas de zonificación que resulten efectivos y acordes a los objetivos de conservación para los que fueron creados el PNIC y el AMM-MS (Hearn et al., 2010; Bessudo et al., 2011).

Declaración de ética: los autores declaran que todos están de acuerdo con esta publicación y que han hecho aportes que justifican su autoría; que no hay conflicto de interés de ningún tipo; y que han cumplido con todos los requisitos y procedimientos éticos y legales pertinentes. Todas las fuentes de financiamiento se detallan plena y claramente en la sección de agradecimientos. El respectivo documento legal firmado se encuentra en los archivos de la revista.

\section{AGRADECIMIENTOS}

Agradecemos al personal del Área de Conservación Isla del Coco por su colaboración en la emisión eficiente de los permisos de investigación otorgados y por asegurar el desarrollo fluido de las actividades. Gracias especiales a los yates privados Alucia y Umbra operados por Alucia Productions, y al personal del yate Argo de la compañía de buceo Undersea Hunter Group por su colaboración incondicional y cordial. Finalmente agradecemos a nuestros patrocinadores: Fundación Amigos de la Isla del Coco (FAICO), la Whitley Fund for Nature (WFN), Conservación Internacional-Costa Rica, la Sandler Family Foundation, y la PEW Fellowship in Marine Conservation.

\section{RESUMEN}

Introducción: Los montes submarinos y las islas oceánicas son conocidos como "puntos calientes" de biodiversidad pelágica, los cuales son utilizados por especies altamente migratorias como corredores biológicos naturales. En el Pacífico Tropical Oriental se han establecido una serie de Áreas Marinas Protegidas (AMPs) cuyo objetivo principal es proteger y manejar los recursos marinos que se encuentran dentro de esta región. Sin embargo, muchas de estas AMPs no incluyen esquemas de protección absoluta, 
y ninguna cuenta con la capacidad de de proteger a las especies altamente móviles una vez que estas se desplazan fuera de sus límites. Objetivos: El objetivo del presente estudio es dar a conocer la primer evidencia de conectividad entre el Parque Nacional Isla del Coco (PNIC) y el monte submarino Las Gemelas ubicado dentro del Área Marina de Manejo Montes Submarinos (AMM-MS) registrada por un tiburón martillo hembra (Sphyrna lewini). Métodos: Entre mayo del 2015 y mayo del 2016 un tiburón martillo y un tiburón zorro (Alopias pelagicus) fueron marcados (V16, Vemco Ltd.) y monitoreados por una serie de receptores acústicos ubicados en el PNIC y en Las Gemelas. Los datos fueron analizados descriptivamente y se calculó el índice de residencia (IR). Resultados: S. lewini realizó viajes de ida y vuelta entre los sitios ubicados dentro del PNIC y el AMM-MS. El mayor número de detecciones registradas por S. lewini ocurrieron en Roca Sucia, y todas las detecciones tuvieron lugar durante las horas de la mañana (7:00 - 12:00 h). S. lewini exhibió una baja residencia ( $\mathrm{IR}=0.02)$ y una presencia periódica en el área de estudio. Se detectó la presencia de $A$. pelagicus en Las Gemelas casi un año después de haber sido marcado en ese mismo lugar. Conclusiones: La presencia periódica de $S$. lewini entre estas dos AMPs, y la presencia de $A$. pelagicus en Las Gemelas sugiere que los movimientos entre las islas oceánicas de la región puede ser más complejo que simples movimientos de "ida y vuelta", donde los montes submarinos juegan un papel importante en el desplazamiento de dichas especies. Nuestros resultados destacan la importancia de aumentar la protección a los montes submarinos y de crear corredores marinos que conecten las islas oceánicas con el fin de promover la conservación de especies altamente migratorias. Aunque la creación de AMPs alrededor de estas formaciones geológicas se ha propuesto como una manera efectiva de conservar y proteger la biodiversidad marina en aguas abiertas, su efectividad es solo parcial. Esta investigación resalta la importancia de estudiar la dinámica espacial de especies altamente migratorias con el objetivo de mejorar el diseño y la efectividad de AMPs ubicadas lejos del continente como es el caso del PNIC y el AMM-MS.

Palabras clave: Pacífico Tropical Oriental, especies altamente migratorias, Áreas Marinas Protegidas, Sphyrna lewini, Alopias pelagicus.

\section{REFERENCIAS}

Arias, A., Pressey, R. L., Jones, R. E., Álvarez-Romero, J. G., \& Cinner, J. E. (2016). Optimizing enforcement and compliance in offshore marine protected areas: a case study from Cocos Island, Costa Rica. Oryx, 50(1), 18-26.

Baum, J., Clarke, S., Domingo, A., Ducrocq, M., Lamónaca, A.F., Gaibor, N., Graham, R., Jorgensen, S., Kotas, J.E., Medina, E., Martinez-Ortiz, J., Monzini Taccone di Sitizano, J., Morales, M.R., Navarro, S.S., Pérez-Jiménez, J.C., Ruiz, C., Smith, W.,
Valenti, S.V. \& Vooren, C.M. (2009). Sphyrna lewini. The IUCN Red List of Threatened Species 2009: e.T39385A10190088.

Bessudo, S., Soler, G. A., Klimley, A. P., Ketchum, J. T., Hearn, A., \& Arauz, R. (2011). Residency of the scalloped hammerhead shark (Sphyrna lewini) at Malpelo Island and evidence of migration to other islands in the Eastern Tropical Pacific. Environmental Biology of Fishes, 91, 165-176.

Cajiao, M. (2008). Aspectos legales relacionados con el Parque Nacional Isla del Coco, Costa Rica. Revista de Biología Tropical, 56(Supl. 2), 207-214.

Clarke, T. A. (1971). Ecology of scalloped hammerhead shark, Sphyrna lewini, in Hawaii. Pacific Science, $25,133-144$.

Cortés, J., \& Blum, S. (2008). Life to $450 \mathrm{~m}$ depth at Isla del Coco, Costa Rica. Revista de Biología Tropical, 56(Supl. 2), 189-206.

Cortés, J., Sánchez-Jiménez, A., Rodríguez-Arrieta, J. A., Quirós-Barrantes, G., González, P. C., \& Blum, S. (2012). Elasmobranchs observed in deepwaters (45-330m) at Isla del Coco National Park, Costa Rica (Eastern Tropical Pacific). Revista de Biología Tropical, 60(Supl. 3), 257-273

Dapp, D., R. Arauz, J. Spotila and M.P. O’Connor. (2013). Impact of the Costa Rican longline fishery on its by catch of sharks, stingrays, bony fish and olive ridley turtles (Lepidochelys olivacea). Journal of Experimental Marine Biology and Ecology, 448, 228-239.

Decreto Ejecutivo No 36452-MINAET. Creación del Área Marina de Manejo Montes Submarinos. Costa Rica. 3 de marzo de 2011 .

Dewar, H., Mous, P., Domeier, M., Muljadi, A., Pet, J., \& Whitty, J. (2008). Movements and site fidelity of the giant manta ray, Manta birostris, in the Komodo Marine Park, Indonesia. Marine Biology, 155, 121-133.

Espinoza, M., Heupel, M. R., Tobin, A.J., \& Simpfendorfer, C. A. (2015). Movement patterns of silvertip sharks (Carcharhinus albimarginatus) on coral reefs. Coral Reefs. 34(3), 807-821.

Hamilton III, W. J., \& Watt, K. E. F. 1970. Refuging. Annual Review of Ecology and Systematics, 1, 263-286.

Hearn, A., Ketchum, J., Klimley, A. P., Espinoza, E., \& Peñaherrera, C. (2010). Hotspots within hotspots? Hammerhead shark movements around Wolf Island, Galápagos Marine Reserve. Marine Biology, 157, 1899-1915.

Hearn, A., Utreras, E., \& Henderson. S. (2010). Informe sobre el estado de los tiburones del Pacífico Este 
Tropical, Vol. 1, Conservación Internacional, Quito, Ecuador, 32 p.

Heidemeyer, M. (2015). Orígenes natales y migratorios de la agregación de tortuga negra (Chelonia mydas agassizii) en el hábitat de alimentación de la Isla del Coco basado en análisis de ADN, bioquímicos $y$ tecnología satelital. (Tesis de maestría). Escuela de Biología, Universidad de Costa Rica, San Pedro, Costa Rica.

Holland, K. N., Wetherbee, B. M., Peterson, J. D., \& Lowe, C. G. (1993). Movements and distribution of hammerhead shark pups on their natal grounds. Copeia, 1993, 495-502.

Hoyos-Padilla, E. M., Ketchum, J. T., Klimley, A. P., \& Galván-Magaña, F. (2014). Ontogenetic migration of a female scalloped hammerhead shark Sphyrna lewini in the Gulf of California. Animal Biotelemetry, 2(1), 17.

Ketchum, J. T., Hearn, A., Klimley, A. P., Peñaherrera, C., Espinoza, E., Bessudo, S., Soler, G., \& Arauz, R. (2014a). Inter-island movements of scalloped hammerhead sharks (Sphyrna lewini) and seasonal connectivity in a marine protected area of the eastern tropical Pacific. Marine Biology, 161, 939-951.

Ketchum, J. T., Hearn, A., Klimley, A. P., Espinoza, E., Peñaherrera, C., \& Largier, J. L. (2014b). Seasonal changes in movements and habitat preferences of the scalloped hammerhead shark (Sphyrna lewini) while refuging near an oceanic island. Marine Biology, 161, 755-767.

Klimley, P. A., \& Nelson, D. R. (1984). Diel movement patterns of the scalloped hammerhead shark (Sphyrna lewini) in relation to El Bajo Espiritu Santo: a refuging central-position social system. Behavioral Ecology and Sociobiology, 15, 45-54.

Klimley, P. A. (1993). Highly directional swimming by scalloped hammerhead sharks, Sphyrna lewini, and subsurface irradiance, temperature, bathymetry, and geomagnetic field. Marine Biology, 117(1), 1-22.

Lavelle, W. \& Mohn, C. (2010). Motion, commotion, and biophysical connections at deep ocean seamounts. Oceanography 23,91-103.

Lizano, O. G. (2012). Rasgos morfológicos alrededor de la Isla del Coco y de sus montes submarinos vecinos, Pacífico de Costa Rica. Revista de Biología Tropical, 60(Supl. 3), 43-51.

Lohmann, K. J., Lohmann, C. M., \& Endres, C. S. (2008). The sensory ecology of ocean navigation. Journal of Experimental Biology, 211(11), 1719-1728.

López-Garro, A., Zanella, I., Martínez, F., Golfín-Duarte, G., \& Pérez-Montero, M. (2016). Illegal fishing at Isla del Coco National Park, Costa Rica. Revista de Biología Tropical, 64(Supl. 1), 249-261.
Meneses, R. (2005). Antecedentes, principios y objetivos del Corredor Marino. Ambientico, 138, 4-5.

MigraMar. (2019). http://migramar.org. Consultada el 4 de mayo del 2019.

Morato, T., Hoyle, S. D., Allain, V., \& Nicol, S. J. (2010). Seamounts are hotspots of pelagic biodiversity in the open ocean. Proceedings of the National Academy of Sciences, 107(21), 9707-9711.

Nalesso, E. (2014). Distribución espacio-temporal de los tiburones martillo, Sphyrna lewini, alrededor de la Isla del Coco (2005-2013), Pacifico Tropical Oriental. (Tesis de Maestría). Centro de Investigación Científica y de Educación Superior, Baja California, México.

Nalesso, E., Hearn, A., Sosa-Nishizaki, O., Steiner, T., Antoniou, A., Reid, A., Bessudo, S., Soler, G., Klimley, A.P., Lara, F., Ketchum, J. T., \& Arauz, R. (2019). Movements of scalloped hammerhead sharks (Sphyrna lewini) at Cocos Island, Costa Rica and between oceanic islands in the Eastern Tropical Pacific. PLoS ONE 14(3): e0213741. https://doi.org/10.1371/ journal.pone. 0213741

Nosal, A. P., Chao, Y., Farrara, J. D., Chai, F., \& Hastings, P. A. (2016). Olfaction Contributes to Pelagic Navigation in a Coastal Shark. PLoS ONE 11(1): e0143758. https://doi.org/10.1371/journal.pone.0143758

Oliver, S. P., Hussey, N. E., Turner, J. R., \& Beckett, A. J. (2011). Oceanic Sharks Clean at Coastal Seamount. PLoS ONE, 6 (3): e14755. https://doi.org/10.1371/ journal.pone.0014755

Peñaherrera-Palma, C., Arauz, R., Bessudo, S., BravoOrmaza, E., Chassot, O., Chinacalle-Martínez, N., Espinoza, E., Forsberg K., García-Rada, E., Guzmán, H., Hoyos, M., Hucke, R., Ketchum, J., Klimley, A.P., López-Macías, J., Papastamatiou, Y., Rubin, R., Shillinger, G., Soler, G., Steiner, T., Vallejo, F., Zanella, I., Zárate, P., Zevallos-Rosado, J. \& Hearn, A. (2018). Justificación biológica para la creación de la MigraVía Coco-Galápagos. MigraMar y Pontificia Universidad Católica del Ecuador Sede Manabí. Portoviejo, Manabí, Ecuador. 80 pp.

Pitcher, T. J., Morato, T., Hart, P. J., Clark, M. R., Haggan, N., \& Santos, R. S. (2008). Seamounts: ecology, fisheries and conservation. John Wiley \& Sons.

Reardon, M., Márquez, F., Trejo, T. \& Clarke, S.C. (2009). Alopias pelagicus. The IUCN Red List of Threatened Species 2009: e.T161597A5460720. http://dx.doi.org/10.2305/IUCN.UK.2009- 2.RLTS. T161597A5460720.en

Rogers, A. D. (1994). The biology of seamounts. In Advances in marine biology. Academic Press.30, 305-350.

Rojas, W., \& Alvarado, G. E. (2012). Marco geológico y tectónico de la Isla del Coco y la región marítima 
circunvecina, Costa Rica. Revista de Biología Tropical, 60(Supl. 3), 15-32.

Rodríguez A. \& Rosero O. (2018). Caracterización y Análisis de la Presión de la Pesca Industrial en el Área de Conservación Marina Cocos y la Zona Económica Exclusiva aledaña de Costa Rica. Amigos Isla del Coco - Faico. San José, Costa Rica. 63 pp.

Rothschild, M. (2005). Corredor Marino: responsabilidad compartida. Ambientico 138, 10-14.

Salinas-de-León, P., Hoyos-Padilla, E. M., \& Pochet, F. (2017). First observation on the mating behaviour of the endangered scalloped hammerhead shark Sphyrna lewini in the Tropical Eastern Pacific. Environmental Biology of Fishes, 100(12); 1603-1608.

Sandin, S. A., Smith, J. E., DeMartini, E. E., Dinsdale, E. A., Donner, S. D., et al. (2008). Baselines and Degradation of Coral Reefs in the Northern Line Islands. Plos One 3: e1548.

Sibaja-Cordero, J. A. (2008). Tendencias espacio-temporales de los avistamientos de fauna marina en los buceos turísticos (Isla del Coco, Costa Rica). Revista de Biología Tropical, 56(Supl. 2), 113-132.

SINAC. (2013). Plan de Manejo del Área Marina de Manejo de Montes Submarinos (AMM-MS), Costa Rica. Área de Conservación Marina Isla del Coco (ACMIC). 102 pp.
Starr, R. M., Green, K., \& Sala, E. (2012). Deep-water fish assemblages at Isla del Coco National Park and Las Gemelas Seamount, Costa Rica. Revista de Biología Tropical, 60(Supl. 3), 347-362.

Torres-Rojas, Y. E., Páez-Osuna, F., Hernández-Herrera, A., Galván-Magaña, F., Aguiñiga-García, S., Villalobos-Ortíz, H., \& Sampson, L. (2013). Feeding grounds of juvenile scalloped hammerhead sharks (Sphyrna lewini) in the south-eastern Gulf of California. Hidrobiología, 726, 81-94.

Wehrtmann, I. S., \& Cortés, J. (2009). Marine Biodiversity of Costa Rica, Central America. Vol. 86. Springer Science \& Business Media.

White, E. R., Myers, M. C., Flemming, J. M., \& Baum, J. K. (2015). Shifting elasmobranch community assemblage at Cocos Island - an isolated marine protected area. Conservation Biology, 29(4), 1186-1197.

Worm, B., Lotze, H. K., \& Myers, R. A. (2003). Predator diversity hotspots in the Blue Ocean. Proceedings of the National Academy of Sciences, 100(17), 9884-9888.

Zanella, I., López-Garro, A., \& Arauz, R. (2009). Caracterización de la pesca del tiburón martillo, Sphyrna lewini, en la parte externa del Golfo de Nicoya, Costa Rica. Revista de Ciencias Marinas y Costeras, 1, 175-195. 